\title{
CASE OF METASTATIC UROTHELIAL CARCINOMA OF THE RETINA AND VITREOUS
}

Katherine E. Whalen, MD, ${ }^{*}$ Ralph C. Eagle, Jr., MD,† Tamara R. Vrabec, MD*

Purpose: To report a rare case of vitreo-retinal metastasis from urothelial carcinoma of the bladder.

Methods: Case report.

Results: A 55-year-old man with a history of bladder cancer developed atypical vitritis and a white fundus mass. Intravenous fluorescein angiography demonstrated connection between the retinal and tumor vasculature consistent with a retinal malignancy. Cytologic analysis of the vitreous sample revealed large, atypical cells with pleomorphic nuclei, mucin vacuoles, and rare mitotic figures. The cells were immunoreactive for cytokeratin markers AE1/AE3, CK7, and CK20 and the urothelial carcinoma marker GATA3. Review of the patient's initial bladder tumor revealed an anaplastic epithelial neoplasm with dyscohesive cells that appeared histologically identical to those in the vitreous biopsy. Despite external beam radiation therapy, the patient's vision declined and the eye became painful and was ultimately enucleated.

Conclusion: Retinal metastasis from systemic adenocarcinoma is an extremely rare occurrence with poor prognosis for vision.

RETINAL CASES \& BRIEF REPORTS 12:177-180, 2018

From the *Department of Ophthalmology, Geisinger Medical Center, Danville, Pennsylvania; and †Department of Pathology, Wills Eye Hospital, Philadelphia, Pennsylvania.

$\mathrm{W}$ e report the first documented case, to our knowledge, of a vitreo-retinal metastasis from a urogenital primary source.

Presented at Verhoeff-Zimmerman Society, San Diego, CA, April 10, 2015; Midwest Ocular Angiography Society, Budapest, Hungary, July 17, 2015; ASRS, Vienna, Austria, August 2015; Wills Eye Intraocular Tumor Symposium, Philadelphia PA, May 29, 2015; The Retina Society, Paris, France, October 10, 2015.

None of the authors has any financial/conflicting interests to disclose.

This is an open-access article distributed under the terms of the Creative Commons Attribution-Non Commercial-No Derivatives License 4.0 (CCBY-NC-ND), where it is permissible to download and share the work provided it is properly cited. The work cannot be changed in any way or used commercially without permission from the journal.

Reprint requests: Katherine E. Whalen, MD, Department of Ophthalmology, Geisinger Medical Center, 100 North Academy Avenue, MS 21-20, Danville, PA 17822; e-mail: trvrabec@ geisinger.edu

\section{Case Report}

A 55-year-old man presented with a 3-month history of increasing floaters without photopsias. Systemic review of systems was positive for anorexia, night sweats, and fatigue. Medical history was significant for chronic hepatitis $\mathrm{C}$, hemochromatosis, and high-grade urothelial carcinoma of the bladder treated with cystoprostatectomy with ureteroileal conduit 4 years earlier. Histopathologic analysis of the bladder tumor demonstrated invasion of the muscularis mucosae but not the muscularis propria with $1 / 11$ + nodes (Stage T1N1M0). The patient received adjuvant chemotherapy with four cycles of carboplatin and Taxol.

Visual acuity on presentation was 20/20-1 in the right eye and 20/20-1 in the left eye. Anterior segment biomicroscopy revealed $1+$ anterior chamber cells and a few keratic precipitates in the right eye. There were 2 to $3+$ retrolental and mid-vitreous cells with snowballs and debris inferiorly. The optic nerve and macula appeared normal. An elevated white, vascularized lesion was present in the inferonasal retinal periphery with surrounding subretinal fluid and satellite lesions (Figure 1A). Examination of the left eye was normal.

Intravenous fluorescein angiography showed neither cystoid macular edema nor vasculitis but demonstrated communication between the retinal and tumor vasculature (Figure 1B). B-scan ultrasonography revealed particulate matter in the vitreous cavity and subretinal space and an area of thickened retina (Figure 1C).

Differential diagnosis included retinal abscess, metastasis, and granuloma. Laboratory test reports including RPR, 


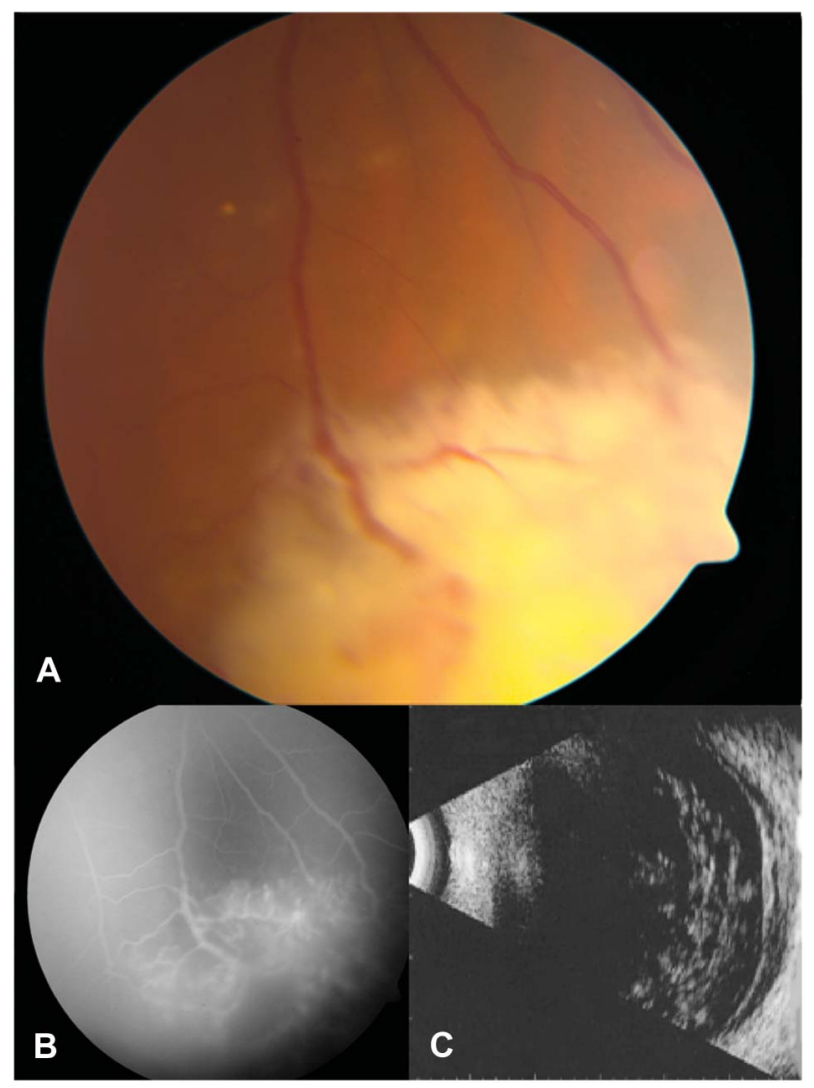

Fig. 1. Initial clinical features. A. The amelanotic fundus mass is remarkable for corkscrew vascularity. B. Intravenous fluorescein angiography demonstrates communication between retinal and tumor vasculature. C. Ultrasonography revealed retinal thickening and intravitreal and subretinal particulate material.

fluorescent treponemal antibody absorption, quantiferon gold, Toxoplasma immunoglobulins $\mathrm{G}$ and $\mathrm{M}$, chest $\mathrm{x}$-ray, Lyme titers, and blood cultures were negative. Computed tomographies of the brain and orbits, chest, abdomen, and pelvis were negative for metastatic disease.

A diagnostic vitrectomy was performed. Histopathologic examination of the vitreous sample revealed sheets and strands of vitreous with numerous dyscohesive cells that were round or oval with significant nuclear pleomorphism and rare mitotic figures. Signet ring cells that contained vacuoles of periodic acid Schiff (PAS), and alcian blue-positive mucin were identified. Immunohistochemical staining (IHC) of the nuclei of the tumor cells was intensely positive for transcription factor GATA3, which is specific for urothelial and breast carcinoma. The cells were also strongly immunoreactive for epithelial markers AE1/AE3 and CK7 and CK20. Coexpression of CK7 and CK20 is a characteristic feature of urothelial carcinoma and excludes breast carcinoma that is only positive for CK7.

S-100 protein showed mild cytoplasmic staining and leukocyte common antigen was negative. Sections from the patient's original bladder tumor were reviewed, and cells in the vitreous biopsy appeared to be identical (Figure 2).

Despite external beam radiation therapy $(3,750$ cGy in 15 fractional doses), the tumor burden increased causing pseudohypopyon, pseudo posterior subcapsular cataract, and dense vitreous opacification (Figure 3). Visual acuity declined to no light perception. Intraocular pressure was $22 \mathrm{mmHg}$. The blind, painful eye was enucleated one year after the onset of visual symptoms. Sections revealed mass of tumor cells in the retina (Figure 2) with no evidence of choroidal involvement. Uveal involvement was detected in the iris stroma and anterior pars plicata. The vitreous and the anterior chambers contained significant quantities of tumor (Figure 4).

\section{Discussion}

Bladder cancer is the second most common genitourinary malignancy, accounting for $7 \%$ of cancer in men and $3 \%$ in women. ${ }^{1}$ Approximately $90 \%$ of epithelial tumors are urothelial carcinomas arising from the transitional epithelium of the
Fig. 2. Histopathology. Sections from the patient's original bladder tumor (A) and cells in the vitreous biopsy (B) appear similar. Both are characterized throughout by dyscohesive cells with significant nuclear pleomorphism consistent with an anaplastic epithelial neoplasm. Arrow indicates "signet ring" cell.
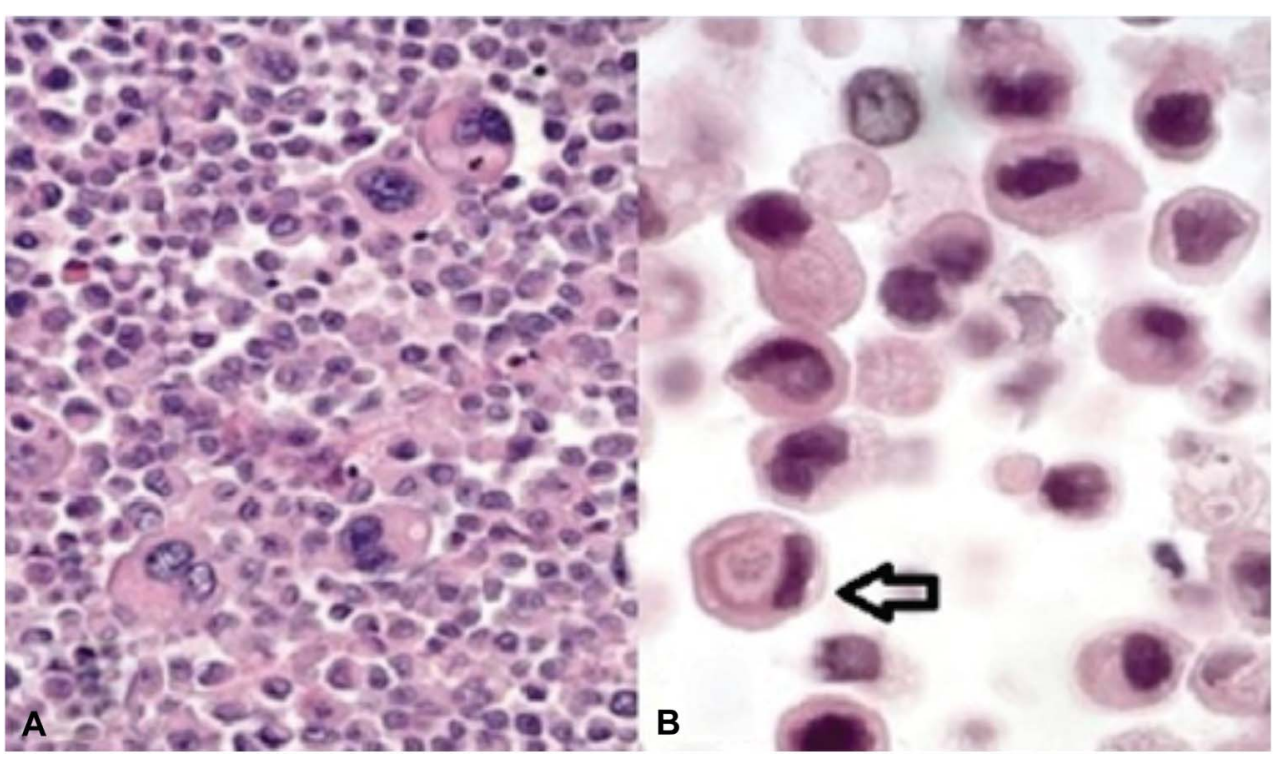


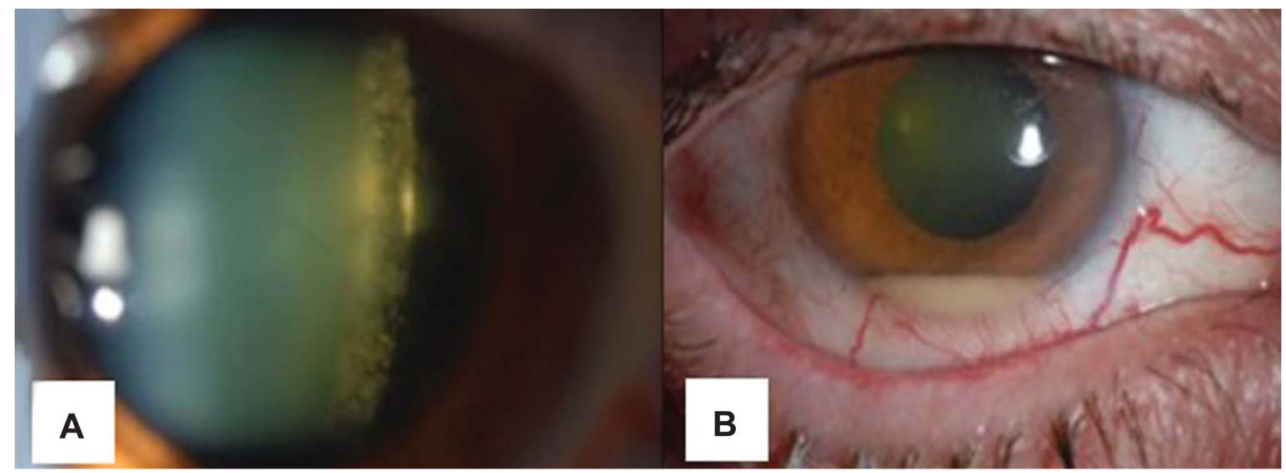

Fig. 3. Tumor cell precipitation on the posterior surface of the lens (A) and in the anterior chamber $(\mathbf{B})$ creates an appearance of pseudo posterior subcapsular cataract and pseudohypopyon.

bladder. High-grade tumors tend to recur locally and have a propensity for invading the bladder wall musculature. The most common sites of metastasis are the lymph nodes, liver, lung, bone, adrenal glands, and intestine. ${ }^{1,2}$ The 5-year survival rate from urothelial tumors confined to the epithelium is 94\% (carcinoma in situ or Stage 0), but declines sharply to $40 \%$ with locally advanced (Stage III) disease. The 5-year survival for those with distant metastases is $5 \% .^{2}$

Ocular metastasis from urothelial carcinoma is extremely rare. Previously reported cases have identified tumors in the orbit or choroid. ${ }^{1,3,4}$ Choroidal metastasis carries a poor prognosis. Levecq et $\mathrm{al}^{3}$ identified a survival rate ranging from 2 months to
7 months. To our knowledge, this is the first reported case of vitreo-retinal metastasis of urothelial carcinoma.

Retinal metastases are rare tumors. A comprehensive case study from 1997 identifying 520 cases of ocular metastasis by the site of eye involvement reported that $88 \%$ of ocular metastases were choroidal, $9 \%$ were to the iris, and $2 \%$ were to the ciliary body, and none were vitreo-retinal..$^{5}$ A review of the recent literature identified only 55 cases of metastatic retinal tumors. The most commonly identified primary tumors were cutaneous melanoma (30.9\%), lung carcinoma (25.5\%), breast carcinoma (18.2\%), and gastrointestinal tumors $(16.4 \%) .{ }^{6}$ The primary tumors were occult in $5.5 \%$ of cases. A recent case

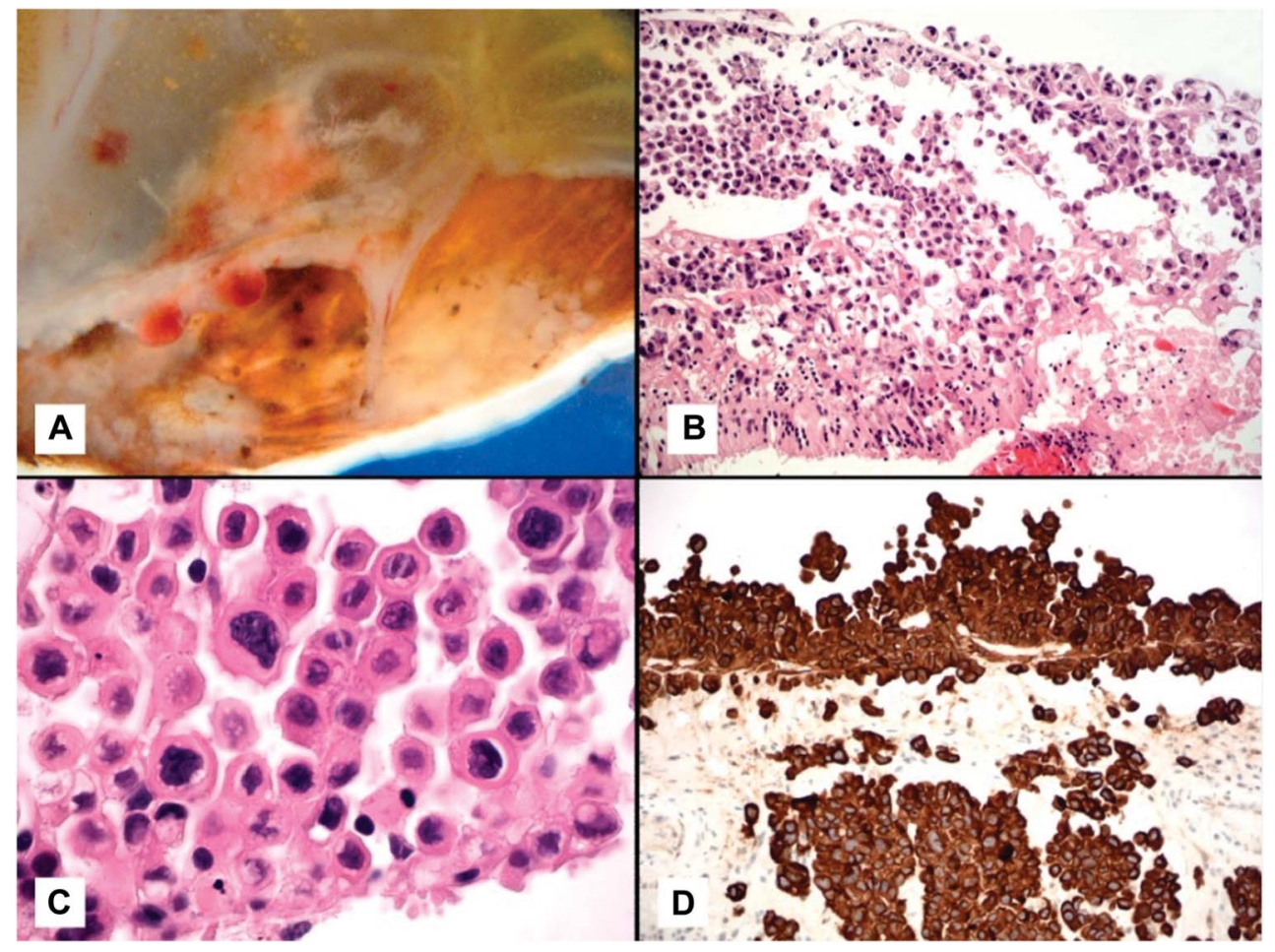

Fig. 4. A. Detached retina in the enucleated eye is thickened and opacified by tumor. Bilobed hemorrhage on the outer surface corresponds to the structure noted on B-scan ultrasound. B. Histopathology discloses diffuse infiltration of retina by round, dyscohesive tumor cells identical to those comprising primary bladder cancer and retinal biopsy (Hematoxylin and eosin, $\times 50$ ). C. Higher magnification of tumor cells shows significant nuclear pleomorphism and scattered mitotic figures (Hematoxylin and eosin, $\times 400)$. D. Tumor cells within retina and on the inner surface of internal limiting membrane are intensely immunoreactive to the cytokeratin marker CK7. Cells also stained with CK20 and GATA3 consistent with urothelial carcinoma (IHC for CK7, ×100). 
series of eight patients with retinal metastasis highlighted the poor prognosis for survival. Five of 8 patients $(62.5 \%)$ died within one month of diagnosis of retinal metastasis. ${ }^{7}$

In summary, we report the first case of metastatic urothelial carcinoma of the retina and vitreous. Unlike previously reported ocular metastatic urothelial carcinomas and retinal metastatic tumors for which prognosis for survival is extremely poor, our patient remains well 23 months after presentation.

Key words: urothelial carcinoma, retinal metastasis.

\section{Acknowledgments}

The authors acknowledge Dr. Carol Shields of the Wills Eye Oncology Service who was instrumental in the care of the patient.

\section{References}

1. Mitsui Y, Arichi N, Inoue K, et al. Choroidal and cutaneous metastasis from urothelial carcinoma of the bladder after radical cystectomy: a case report and literature review. Case Rep Urol 2014;2014:491541.

2. Gupta R, Paner GP, Amin MB. Neoplasms of the upper urinary tract: a review with focus on urothelial carcinoma of the pelvicalyceal system and aspects related to its diagnosis and reporting. Adv Anat Pathol 2008;15:127-139.

3. Levacq L, De Potter P, Godfraind C, et al. Choroidal metastasis from carcinoma of the bladder. Retina Cases Brief Rep 2007;1:251-253.

4. Nabi G, Dadeya S, Dogra PN, Lal H. Eye metastasis form urothelial tumors. Int Urol Nephrol 2002;34:51-54.

5. Shields CL, Shield JA, Gross NE, et al. Survey of 520 eyes with uveal metastases. Ophthalmology 1997;104:1265-1276.

6. Eagle RC. Urothelial carcinoma metastatic to the retina diagnosed by vitreous biopsy. Verhoeff-Zimmerman Society Meeting; San Diego, CA, April 9-12, 2015.

7. Shields CL, McMahon JF, Atalay HT, et al. Retinal metastasis from systemic cancer in 8 cases. JAMA Ophthalmol 2014;132:1303-1308. 